\title{
Computational Design of Potential Binder Protein for SARS-CoV-2 Spike RBD through A Novel Deep Neural Network Based-Protein Outpainting Algorithm
}

\author{
Bingya Duan \\ School of Electronic, Electrical and Communication \\ Engineering, University of Chinese Academy of Sciences, \\ Beijing China \\ duanbingya@ucas.ac.cn
}

\begin{abstract}
COVID-19 caused by SARS-CoV-2 is seriously endangering the health of all human beings. There is an urgent need for drugs that can inhibit the replication and propagation of the virus. Traditional macromolecular drugs have long discovery and development cycles and high experimental costs, which can't give rapid response to new viruses.

Through computational protein design method, scientists have designed binder proteins with high affinity for the RBD of SARSCoV-2 spike protein which can effectively inhibit virus replication. However, traditional computational protein design methods rely heavily on human experience and domain knowledge of protein design, and the protein design workflow is too complicated to be widely accepted and used in academia and industry.

Based on previous work in the field of deep neural network protein structure prediction and protein design, we developed a novel protein outpainting method that can generate the remaining part of the protein based on a given hot spot motif and complete the entire protein. This method can generate stable protein scaffold which can support the functional hot spot motif, resulting in a protein with excellent thermal stability and developability. We tested this method in a drug discovery project with the aim of designing new SARS-CoV-2 inhibitors. Several proteins are obtained which are predicted to be stable and may have high affinity for the RBD of the SARS-CoV-2 spike protein. Although they have not been verified by wet-lab experiments, we believe that these proteins have great potential to be developed into effective drugs for the treatment of COVID-19. The protein outpainting algorithm proposed in this paper has great advantages over traditional protein design methods. It can be applied to many fields that require the design of functional proteins, such as protein drug design, enzyme de novo design, vaccine design, etc. The method will play an important role in reducing the cost of experiments, shortening the research and development period, and improving the successful rate of biological research and development.
\end{abstract}

Permission to make digital or hard copies of all or part of this work for personal or classroom use is granted without fee provided that copies are not made or distributed for profit or commercial advantage and that copies bear this notice and the full citation on the first page. Copyrights for components of this work owned by others than ACM must be honored. Abstracting with credit is permitted. To copy otherwise, or republish, to post on servers or to redistribute to lists, requires prior specific permission and/or a fee. Request permissions from permissions@acm.org.

BIBE2021, July 20-22, 2021, Hangzhou, China

(C) 2021 Association for Computing Machinery.

ACM ISBN 978-1-4503-8929-7/21/07 ..\$15.00

https://doi.org/10.1145/3469678.3469685
Yingfei Sun*

School of Electronic, Electrical and Communication

Engineering, University of Chinese Academy of Sciences, Beijing China

yfsun@ucas.ac.cn

\section{CCS CONCEPTS}

- Applied computing; • Life and medical sciences; • Bioinformatics;

\section{KEYWORDS}

Protein design, Protein outpainting, Deep learning, Rosetta, Protein structure prediction

\section{ACM Reference Format:}

Bingya Duan and Yingfei Sun. 2021. Computational Design of Potential Binder Protein for SARS-CoV-2 Spike RBD through A Novel Deep Neural Network Based-Protein Outpainting Algorithm. In The Fifth International Conference on Biological Information and Biomedical Engineering (BIBE2021), July 20-22, 2021, Hangzhou, China. ACM, New York, NY, USA, 8 pages. https://doi.org/10.1145/3469678.3469685

\section{INTRODUCTION}

As of February 10, 2021, COVID-19 has infected 106 million people worldwide and caused 2.3 million deaths, which severely impaired human health and caused huge economic losses [1]. COVID-19 is caused by SARS-CoV-2, a novel coronavirus. There have been many studies on its pathogenic mechanism [2-5]. One of the mechanisms $[4,5]$ is that the interaction between the receptor binding domain (RBD) of the spike protein and the human ACE2 (angiotensinconverting enzyme 2) protein can mediate the process of membrane fusion, which can help the virus to enter the human cell and then infect the human body. The use of macromolecule drugs to block the interaction between the two proteins are very useful therapeutic method [6]. To target the interaction between S protein RBD and ACE2, various types of treatments have been reported, including vaccines [6], peptides [7-9], antibodies [10-12], mini proteins [11], etc.

Baker group has reported a mini-protein discovered by the computational protein design method which can bind to the $S$ protein RBD with KD of pM level and has good antiviral activity [11], which proves that computational protein design is a very effective method for discovering high-affinity binder for target protein. However, traditional computational protein design methods rely heavily on human protein design experience and domain knowledge. The design workflow is extremely complicated, which is not conducive to the large-scale promotion and popularization of protein design method in academia and industry.

In recent years, protein structure prediction and protein design based on deep neural networks are two emerging research fields 
with rapid development. Many advanced algorithms of deep learning have been applied to the field of computational biology. Protein structure prediction aims to solve the problem of predicting the three-dimensional structure of the protein given amino acid sequence of the protein, that is, predicting the structure from the sequence. A lot of development in protein structure prediction using deep learning method has emerged, such as trRosetta [10], AlphaFold [13, 14] and AlphaFold2 [15]. For more information, please refer other review article $[14,16]$. Protein design aims to solve the problem of finding the amino acid sequence that can stably fold into the target structure given topology structure of protein, that is, predicting the sequence from the structure. Computational protein design benefits a lot from the advancement of deep learning, including the use of deep neural network to generate new protein sequences [15] and structures [17], protein hallucination [18], trDesign [19], etc.

Image inpainting/outpainting is a research field of computer vision (Figure 1A). In recent years, people have developed many image inpainting/outpainting methods based on deep neural networks [20-22]. On the basis of previous protein design work and inspired by the problem of image inpainting/outpainting, we developed a new "protein outpainting" method (Figure 1B) which opened up a new way for function protein design based on hot spot motif.

trRosetta [10] is a previously developed method for protein structure prediction (Figure 2A). Given the amino acid sequence of protein, homologous sequences are searched and aligned to get the multiple sequence alignment (MSA) result. Based on co-evolution information, ResNet [23] is used to predict the distance and angle between residue pairs. The distribution of distance and angles can then be used to construct and predict the three-dimensional structure of the protein. Later, the Baker group developed a protein hallucination method based on trRosetta [10] (Figure 2B). The basic idea is to use MCMC method to optimize the input sequence to increase the difference of residue pair distance and angle distribution between the prediction and the background. The KL divergence increases in the optimization progress to result in a protein with ideal protein characteristics and stable folding.

Based on the above two methods, this paper proposes a new protein outpainting method using fixed hot spot motif for protein generation (Figure 2C). The input is the fixed hot spot motif that we want to complete. When performing sequence optimization, we fixed the sequence of the hot spot motif and modified the optimization objective function so that the fixed motif the newly generated protein is kept to the same as the input, while the difference between the remaining parts of the protein and the background distribution is as large as possible. Please refer to the method section for more details. Using this new algorithm, we designed promising proteins with antiviral activity blocking the interaction between $S$ protein of SARS-CoV-2 and human ACE2.

\section{RESULTS}

\subsection{Analysis of Important Residues of the Interaction Between SARS-CoV-2 S Protein RBD and Human ACE2 Protein}

The structure of the SARS-CoV-2 and human ACE2 protein complex is shown in Figure 3A. There is an alpha helix on the interface of

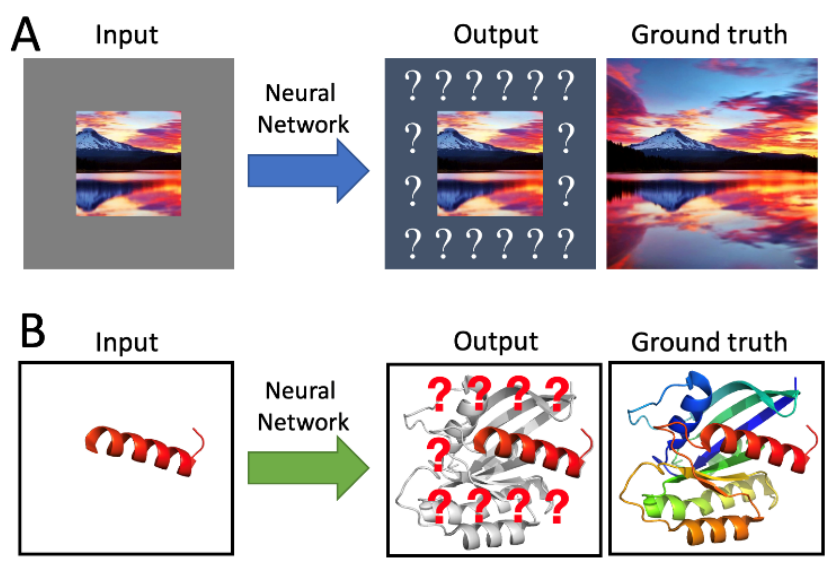

Figure 1: Schematic Diagram of conceptual work Frame for Image Outpainting [20] (A) and Protein Outpainting (B).

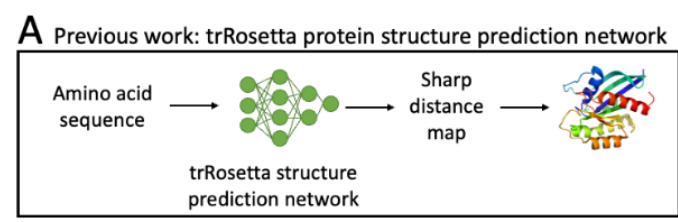

B Previous work: protein design by network hallucination

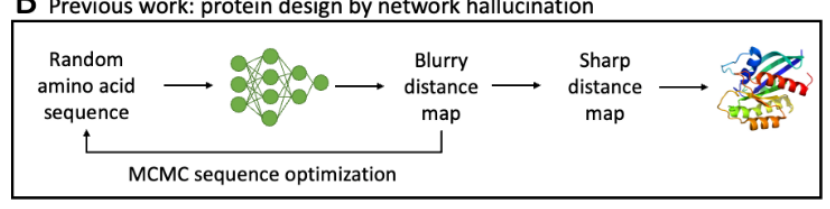

C This work: fixed motif protein outpainting by network hallucination

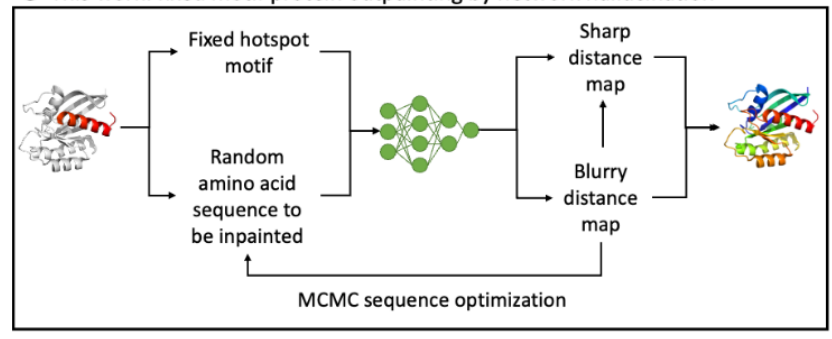

Figure 2: Schematic Diagram of the Principle of the Previous Trrosetta Protein Structure Prediction Method (A), Trrosetta-Based Hallucination Protein Design Method (B), and the New Protein Hallucination-Based Protein Outpainting Method (C) Proposed in This Paper.

ACE2 which plays an important role in the cognization of the two proteins. It has been reported that the synthesized SBP1 peptide (sequence: IEEQAKTFLDKFNHEAEDLFYQS) isolated of residues 21 to 43 from ACE2 protein can bind to $S$ protein with $\mathrm{KD}$ of $47 \mathrm{nM}$ [7], which is sufficient to block the binding of $S$ protein RBD and ACE2. Based on this result, we believe that intercepting this alpha helix and stabilizing it with a new protein scaffold can lead to stronger 

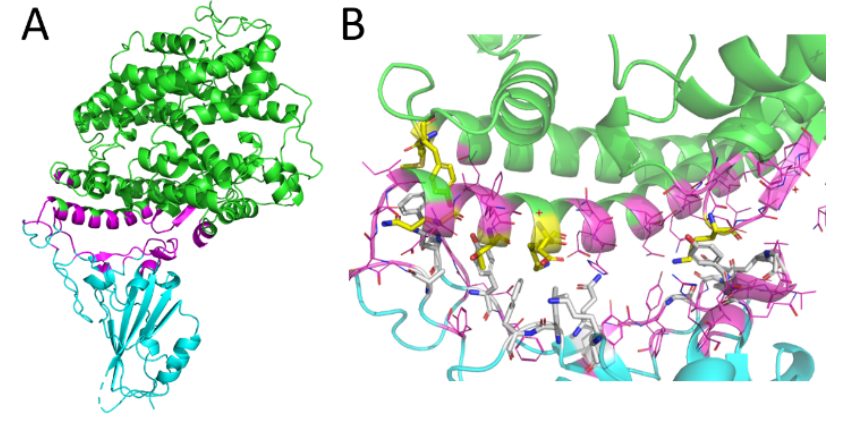

Figure 3: The Overall Structure of the SARS-CoV-2 Spike Protein RBD and Human ACE2 Complex (A) and the Important Residues on the Interface (B). PDB ID: 6M0J.

binding and achieve stronger inhibition of the binding of RBD and ACE2.

In order to analyze the binding property more quantitatively, we used the InterfaceAnalyzer tool [24] in Rosetta [25] to analyze the residues on the binding interface and clarify the contribution of different residues to the binding. The positions of important residues on the protein are shown in Figure 3B.

Human ACE2 is represented in green, spike protein RBD is represented in blue, and the interface between the two proteins is represented in magenta. In Figure 3B, the residues on the ACE2 protein that contribute more than 0.5 REU (Rosetta energy unit) to the binding free energy are represented by yellow sticks, and the residues on the spike protein RBD that contribute more than $0.5 \mathrm{REU}$ are represented by white sticks. The picture is generated using Pymol [26].

We found that the binding free energy between ACE2 protein and S protein RBD was -37.88 REU (Rosetta Energy Unit), of which ACE2 protein contributed $-18.86 \mathrm{REU}$ and S protein RBD contributed -19.02 REU. The ratio of the contribution of different residues to the binding free energy is shown in Figure 4. According to the calculated results, we found that the residues on the ACE2 protein that contribute most to binding are: $353 \mathrm{~K}, 31 \mathrm{~K}, 27 \mathrm{~T}, 30 \mathrm{D}, 82 \mathrm{M}$. The contribution of a single residue of $353 \mathrm{~K}$ accounts for $26 \%$ of total energy. The residues on the RBD of SARS-CoV-2 S protein that contribute most to binding are: 486F, 417K, 502G, 456F. A single residue of $486 \mathrm{~F}$ contributes $16 \%$ of total energy. The value of each residue's contribution to the free binding energy is shown in Table 1.

Based on the results of the energy decomposition of binding interface residues, the contribution of residues 19-42 of ACE2 to the binding energy is $-8.06 \mathrm{REU}$, accounting for $42.7 \%$ of the total binding energy contribution from ACE2.

\subsection{The Workflow of Protein Outpainting Starting From Hotspot Motifs}

In order to block the binding of S protein RBD to ACE2, we propose to design a new protein starting from the 19-42 residue alpha helix of ACE2 to stabilize this alpha helix and enhance the affinity with $S$ protein.
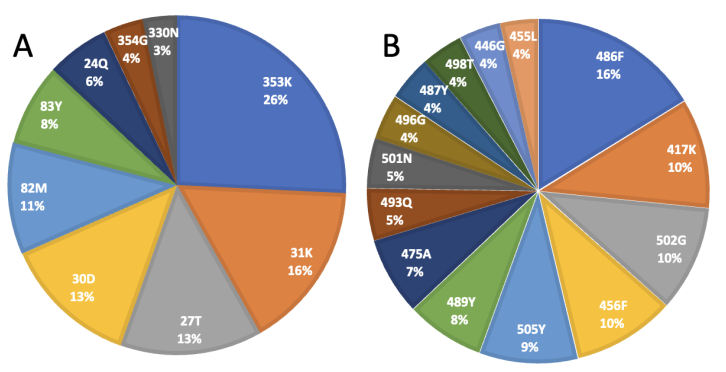

Figure 4: Residue Number and Binding Free Energy Contribution Ratio of Interface Residues from ACE2 (A) and S Protein RBD (B). Only Residues Whose Binding Free Energy Exceeds 0.5 REU are Shown.

Table 1: The contribution of different residues on the binding interface of $h A C E 2$ and $S$ protein $R B D$ to the binding free energy. Only residues whose binding free energy exceeds 0.5 REU are shown.

\begin{tabular}{lll}
\hline Protein & Position number & ddG_contribution (REU) \\
\hline hACE & $353 \mathrm{~K}$ & -4.27 \\
hACE & $31 \mathrm{~T}$ & -2.66 \\
hACE & $27 \mathrm{~T}$ & -2.26 \\
$\mathrm{hACE}$ & $30 \mathrm{D}$ & -2.15 \\
$\mathrm{hACE}$ & $82 \mathrm{M}$ & -1.77 \\
hACE & $83 \mathrm{Y}$ & -1.31 \\
hACE & $24 \mathrm{Q}$ & -0.99 \\
hACE & $354 \mathrm{G}$ & -0.63 \\
hACE & $330 \mathrm{~N}$ & -0.54 \\
S protein RBD & $486 \mathrm{~F}$ & -2.79 \\
S protein RBD & $417 \mathrm{~K}$ & -1.78 \\
S protein RBD & $502 \mathrm{G}$ & -1.71 \\
S protein RBD & $456 \mathrm{~F}$ & -1.67 \\
S protein RBD & $505 \mathrm{Y}$ & -1.60 \\
S protein RBD & $489 \mathrm{Y}$ & -1.27 \\
S protein RBD & $475 \mathrm{~A}$ & -1.26 \\
S protein RBD & $493 \mathrm{Q}$ & -0.84 \\
S protein RBD & $501 \mathrm{~N}$ & -0.81 \\
S protein RBD & $496 \mathrm{G}$ & -0.74 \\
S protein RBD & $487 \mathrm{Y}$ & -0.73 \\
S protein RBD & $498 \mathrm{~T}$ & -0.68 \\
S protein RBD & $446 \mathrm{G}$ & -0.67 \\
S protein RBD & $455 \mathrm{~L}$ & -0.62 \\
\hline
\end{tabular}

As shown in Figure 5, the entire process is mainly divided into the following steps:

(1) Get sequence and structure of the ACE2 protein with target motif.

(2) Isolate the motif to be fixed in new protein with residue 19-42 from ACE2.

(3) Set the initial sequence for optimization. The sequence has a fixed motif (19-42 from ACE2) and random amino acids for 


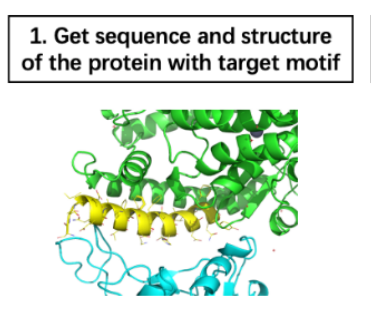

2. Isolate the motif to be fixed in new protein

\begin{tabular}{|c|}
\hline $\begin{array}{c}\text { 3. Initial seq: fixed motif + } \\
\text { random aa for remaining part }\end{array}$ \\
\hline >initial_seq \\
STIEEQAKTFLDKFNHEAE \\
DLFYQQESFPTPSYSNEL \\
HAHDGYMQTNQNRKA \\
AWIKIQGIVGE...
\end{tabular}

4. Optimized through protein inpainting model

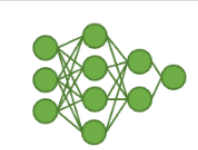

5. Generated new protein

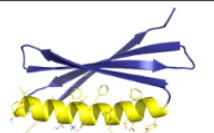

Figure 5: The Flow Chart of Completing and Generating A New Protein from The Hot Spot Residue Motif. The Motif from ACE2 that Needs to Be Fixed Is Indicated in Yellow.

the remaining part. The length is chosen from 50, 60, 70,80, 90 and 100 amino acids.

(4) Optimized the sequence through deep neural network-based protein outpainting model.

(5) Get the optimized new protein, manually inspect and analyze the protein structure.

\subsection{Optimization of the Objective Function in the Process of Protein Outpainting Based on Deep Neural Network}

In the process of protein generation and optimization, the change of the objective function value is shown in Figure 6. It can be seen that in the iterative process of 40,000 steps, the three objective functions fluctuate greatly at the beginning of the iteration (before 10,000 steps), but the overall trend of decreasing is in line with expectations. Among the three objective functions, background_function_value describes the difference between the remaining part of the protein and the ideal protein, which can eventually reach about -4 3; fixed_motif_function_value describes the difference between the fixed motif part of the protein and the structure of the target protein, which can eventually reach $5 \sim 6$; the sum of the two total_function_value can reach about $1 \sim 2$ in the end.

\subsection{The Result of Protein Outpainting Based on Deep Neural Network}

Starting from the alpha helix of residues 19-42 of ACE2, different total length of proteins $(50,60,70,80,90$ and 100) are tried. We used the protein outpainting model to generate 6 different proteins, one protein for each length. As shown in Figure 7, we compared the generated structure with the ACE2-alpha helix-WT structure.

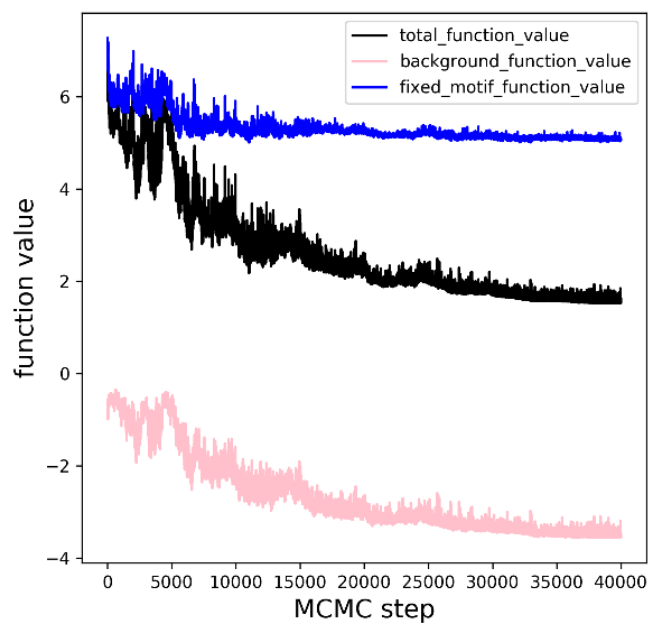

Figure 6: The Value Change of Three Objective Functions Total_Function_Value, Background_Function_Value, and Total_Function_Value in the MCMC Optimization Process.
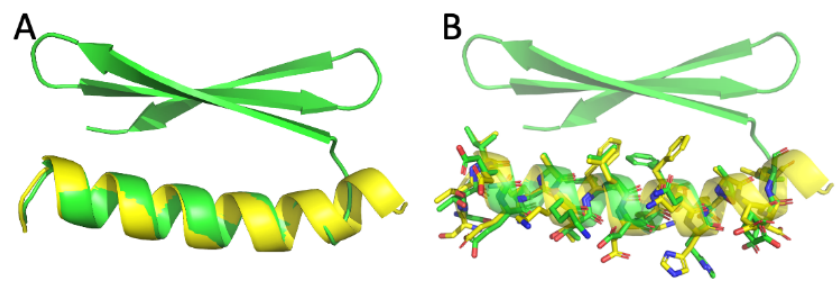

Figure 7: Comparison of the Generated Protein Structure (Length $=50$ ) with the ACE2-alpha Helix-WT Structure.

When the total protein length is 50 , the generated protein structure is alpha helix plus 3 strands of beta sheet. The new protein has a high degree of overlap with the target motif backbone (Figure 7A), and the degree of overlap of the residue side chain conformation is also high (Figure 7B), between which the heavy atom RMSD is 0.943 angstrom. This indicates that the newly generated protein may interact well with the $S$ protein RBD.

The green part is the newly generated protein, and the yellow part is the target alpha helix motif. (A) After superimposed with the target motif, the overlap degree is very high, with an RMSD of 0.943 angstrom. (B) Comparison of side chain conformation of residues 19-42. The amino acid side chain conformation of new protein has a high degree of overlap with the WT conformation.

In addition to the binding affinity with the $S$ protein RBD, whether the new protein itself can fold stably and correctly is also very important for its physiological functions. We analyzed the structure of the newly generated protein as shown in Figure 8. Figure $8 \mathrm{~A}$ shows that the distribution of positive and negative charges on the surface of the protein is relatively uniform which implies that it is not prone to aggregation and can exist stably. Figure $8 \mathrm{~B}$ shows that the protein surface exposed to the solution outside of the beta sheet of the protein is polar residues $(H, T, D$, $\mathrm{K}$, etc.), which is conducive to the stability of the protein in the 

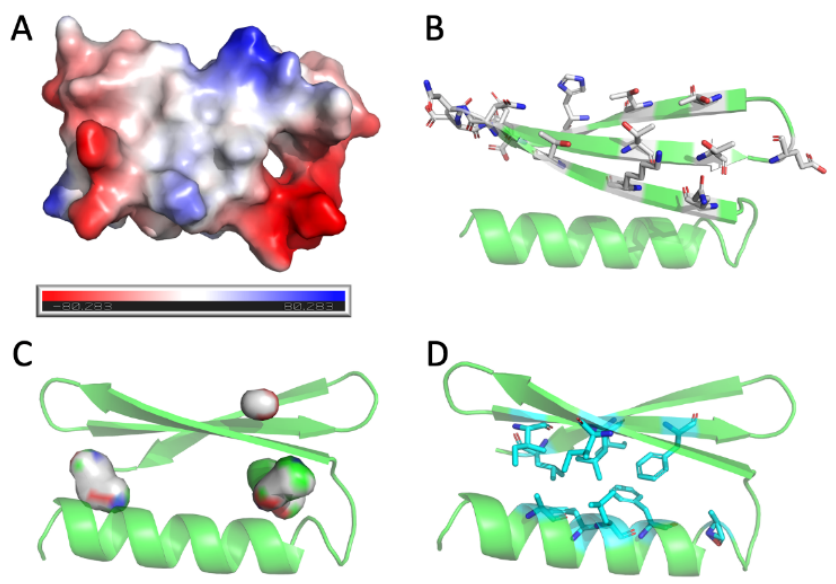

Figure 8: The Structure of the Newly Generated Protein (Length $=50)$.

solution and increases the solubility. Figure $8 \mathrm{C}$ shows that internal packing of the protein is not very tight and cavity exists. This may be a risk point and hint us the direction for further design optimization. Figure 8D shows that there are many hydrophobic and aromatic amino acids (F, L, A, I, etc.) inside the protein, forming good hydrophobic packing, which is good for protein folding and forming stable tertiary structure.

(A) Schematic diagram of protein electrostatic potential. Calculated and rendered with Pymol. (B) The protein surface polar residues exposed to the solution outside the beta sheet. (C) The cavity inside the protein. (D) Good packing of hydrophobic and aromatic residues is formed inside the protein.

\subsection{The Influence of the Total Protein Length on the Results}

We tested the effect of the total protein length of 50, 60, 70, 80, 90 and 100 on the structure of the generated protein. From an intuitive point of view, as shown in Figure 9, the topological structure of the protein generated when the total length is different, and the RMSD of the heavy atom $s$ also different. When the length is 50 amino acids (Figure 9A), the generated protein is a combination of alpha helix and 3 beta sheets; when the length is 60 amino acids (Figure 9B), the generated protein is two bundles of alpha helix; when the length is 70 amino acids (Figure 9C), the generated protein is a combination of alpha helix and 3 beta sheets, and the beta sheet is tilted to one side; when the length is 80 amino acids (Figure 9D), the generated protein is alpha helix and 4 beta sheets; when the length is 90 amino acids (Figure 9E), the resulting protein is three bundles of alpha helix; when the length is 100 amino acids (Figure 9F), the resulting protein is a combination of alpha helix and 5 beta sheets, and the beta sheet is tilted to one side. The most similar protein to the target motif is the protein generated when the length is 100 (Figure 9F), with heavy atom RMSD of 0.769 angstrom. Though the hot spot motif is the most similar, the overall structure of the protein may not be compensated enough to stably fold into 3D
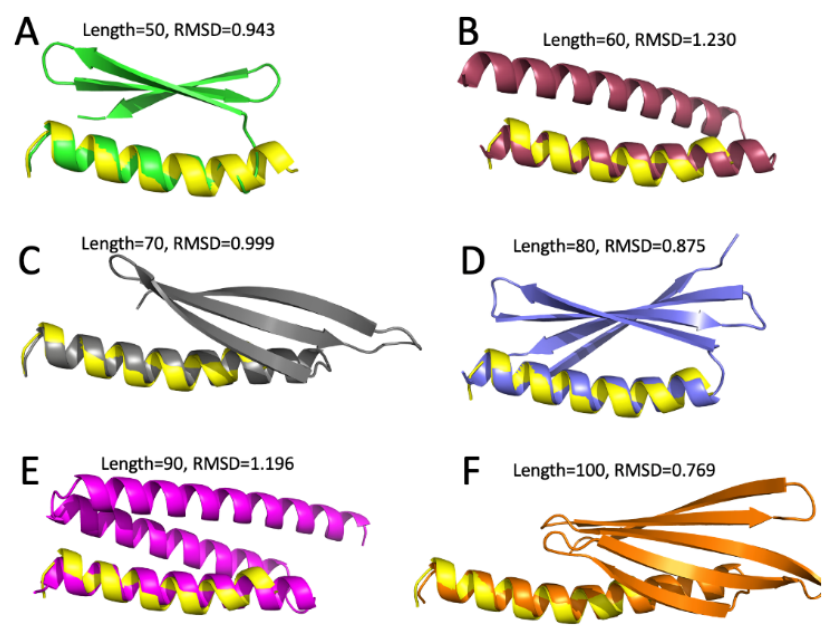

Figure 9: The Topological Structure of Generated Proteins When the Total Length Is Different. The Target Motif Is Shown in Yellow. The Total Length of Each Protein and the Rmsd Value of the Heavy Atom Between the Generated Protein and the Target Motif Are Marked on the Top of the Protein. The Unit of Rmsd is Angstrom.
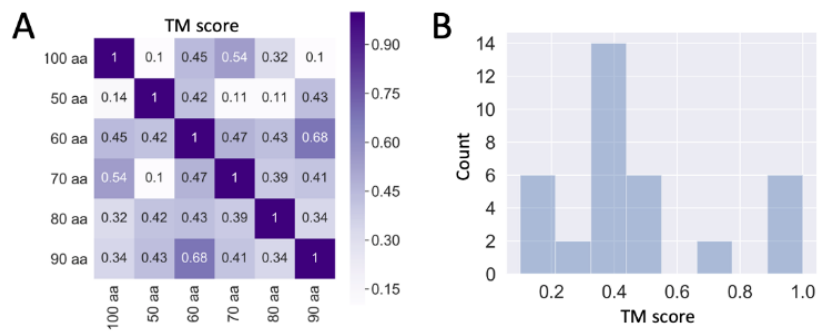

Figure 10: The Heatmap of TM Score Between ProteinProtein Pairs (A) and the Histogram of All TM Scores (B) for Proteins Produced with Different Total Length.

structure since part of the alpha helix is exposed to the solvent without interaction of other parts of the protein (Figure 9F).

In order to quantitatively compare the differences between the topological structures of the generated proteins, we calculated the TM score [26] between the protein-protein pairs. Heat map (Figure 10A) and histogram (Figure 10B) are plotted. TM score is widely used to measure the similarity of two protein structures, especially in protein structure prediction competition [27]. The TM score of most generated protein-protein pairs is less than 0.5 , which means there is a relatively large structural difference, consistent with the result in Figure 9. This suggests that we can set different total protein lengths when generating protein to diversify the protein structure and increase the success rate of protein design. 


\section{DISCUSSION}

\subsection{Advantages of New Proteins Generated Based on Deep Learning Methods Over Antibodies}

Antibody is a widely used macromolecular drug with many good properties, such as high affinity with target protein, high potency, produced by biotechnology and good compatibility with human body. Compared with antibodies, the main advantages of the proteins we designed through deep learning methods are:

(1) Highly stable. The protein can withstand high temperatures above 90 degrees without degeneration, which is conducive to storage and transportation

(2) Can be expressed in E. coli with high expression yield, low cost and simple CMC process.

(3) Small molecular weight means high concentration administration can be achieved, suitable for cocktail therapy. Strong tissue penetration and short metabolic half-life means it will be suitable for antibody-conjugated drugs

(4) Simple structure makes it suitable for making multivalent drugs without the tedious design and process optimization process of multivalent antibodies

\subsection{Advantages and Disadvantages of Protein Completion Methods Based on Deep Learning}

Compared with traditional protein design, deep learning-based methods have the following advantages:

(1) A new protein is generated by the deep neural network without relying on any human experience and knowledge. This method avoids the complicated protein design process and can be easily applied in large scale and automation

(2) The method can generate complex protein structures that humans cannot imagine

(3) The model can generate sequence and structure at the same time

(4) The generated protein is similar to the ideal protein in stability and foldability

(5) The method is of high computational efficiency, which avoids the massive computational resources consumed by the MC simulated annealing method in the traditional method when sampling and optimizing the huge sequence space

The main flaws may include:

(1) The diversity of generated protein may be low. The result may fall into local optimal structure learned by the neural network

(2) We need to design and optimize different objective functions according to different application scenarios

(3) The generated protein may not be able to achieve the desired goal directly in one step, and it needs to be further optimized by other steps, such as further protein design, relax, etc.

\subsection{Application Area of This Method in the Future}

The method proposed in this paper will find broad applications in many fields such as biotechnology, pharmaceutical industry, biosensors, industrial enzyme design, vaccine design, etc., and greatly accelerate the development of corresponding fields. Using this method, we can do the following things: 1 . Non-antibody high-affinity binder protein design with fixed hot spot motif; 2. Cytokine mimics design with specific protein interface; 3 . De novo enzyme design with fixed catalytic residue; 4 . Vaccine antigen design with specific epitope.

\subsection{Limitation of the Work}

(1) The binding affinity of the generated protein with the $S$ protein RBD has not been quantitively evaluated. Later, we can relax the protein by constraining key interactions and then use Rosetta to score the binding affinity or perform MD simulations and calculate the binding energy through MM-PBSA/GBSA method [28].

(2) The folding stability of the generated protein has not been quantitively evaluated. This can be done by biased forward folding [29] using Rosetta or MD simulation.

(3) Due to the limitation of computing resources, combinations of different parameter were not tested. The parameters for model optimization include different motif positions, more protein lengths, etc., which will affect the result and quality of the generated protein.

\section{CONCLUSION}

Through improving the previous protein hallucination method based on trRosetta, we proposed and implemented a novel protein outpainting algorithm. It can be used to generate the remaining part of the protein given a continuous or discontinuous hot spot motif and outpaint the entire protein, with a stable scaffold to support the target functional hot spot motif. We tested this method in the case of designing SARS-CoV-2 inhibitors and successfully designed several proteins which were predicted to be stable and may have high affinity for the RBD of the SARS-CoV-2 spike protein. Our prediction results show that the generated proteins have good stability, affinity and structural diversity, and may have good potential in the treatment of SARS-CoV-2. This protein outpainting algorithm has great advantages over traditional computational protein design methods. It is expected to be widely applied in the fields of macromolecular drug design, de novo enzyme design, vaccine design, etc., to help us improve the accuracy of protein design, reduce experimental costs, shorten $R \& D$ cycle and improve $R \& D$ efficiency.

\section{METHODS}

\subsection{TrRosetta Protein Structure Prediction}

The problem of protein structure prediction is to predict the $3 \mathrm{D}$ structure of the protein when the protein sequence is known. At present, the most representative methods are AlphaFold [13] and AlphaFold2 [15], etc., which can make full use of co-evolution information combined with deep neural networks to generate spatial 
constraints and reduce phase space search, which greatly helps protein structure modeling. At the end of 2019, Baker team proposed trRosetta, which utilized many advances in deep learning and combined with Rosetta modeling software to greatly accelerate protein structure prediction. A particularly important point is that the team found even for many artificial proteins previously designed from scratch which have no homologous sequences, reliable structures can still be predicted with only a single sequence as input using trRosetta.

The input of trRosetta is the MSA information of the sequence. After passing through ResNet [23] neural network, the output is given as the distribution of $\mathrm{d}, \theta, \omega, \psi$ between pairs of all residues. What trRosetta model learns is the relationship between protein sequence and structure. The representation of this knowledge is very different from traditional methods. In traditional de novo protein design, knowledge is embodied in the energy function and sampling method of protein design software (such as Rosetta [25]), while in deep neural networks, the knowledge is distributed among millions of network parameters.

\subsection{Protein Hallucination Method}

First, a random sequence is input to trRosetta's neural network. The distance and angle distribution predicted for these random sequences is different from that of the real protein and is a blurry distance matrix. In order to obtain a new sequence that can be folded into a protein from a random sequence, sequence-level MCMC annealing is used, which means we randomly pick an amino acid and mutate it to other types. The optimized objective function is to maximize the KL divergence. In the optimization process, the KL divergence of the distance and angle distribution between the predicted and the background is as different as possible, making the model biased to search for structures that are as different as possible from an average structure in the database. After 40,000 iterations, the optimized sequence has a very high KL divergence after 40,000 steps, and trRosetta is used to directly generate the scaffold structure.

\subsection{Detailed Description of Protein Outpainting Algorithm Based on Deep Neural Network}

As shown in Figure 11, our goal is to generate a new protein based on the given fixed motif. The remaining part of the protein is imagined by the deep learning model, and the structure and sequence are generated at the same time. In the traditional protein design process, first we have a protein scaffold and then design the protein sequence based on the scaffold. In the de novo protein design process based on deep learning, the difference is that the sequence and structure of the protein are generated at the same time, without human intervention. The flow chart is as following: first we set the length of the total sequence of the protein (such as 100 amino acids). In addition to the fixed motif part, we randomly specify a sequence, and use trRosetta as a starting point to make predictions to obtain $\mathrm{d}, \theta, \omega, \psi$, the distance and angle distribution of 4 parameters. Next, we define the objective function of the fixed motif part and the remaining part of the protein and try to make the KL divergence between the distribution of the fixed motif part of the generated

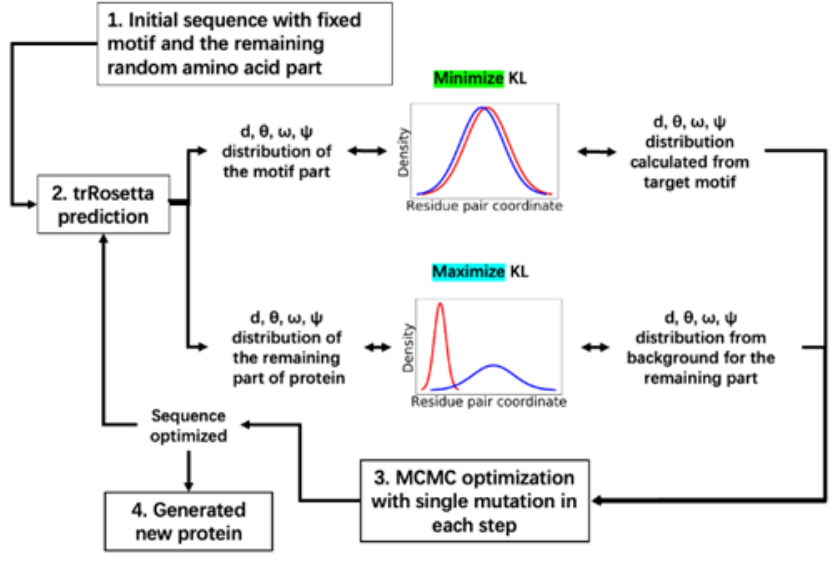

Figure 11: Flowchart of Deep Neural Network Based Fixed Motif Protein Outpainting Algorithm.

protein and the distribution of the initial target motif as small as possible in the optimization process. The KL divergence between the distribution of the rest part and the background noise is optimized to be as large as possible, aiming that the predicted protein characteristics are more sharp and closer to the real protein. In the optimization process, for each iteration we will randomly select a position of an amino acid and randomly mutate it to one of 20 amino acids. After the mutation, the 4 parameters $\mathrm{d}, \theta, \omega, \psi$ are calculated using to trRosetta and the value of the objective function are obtained. If the value of the objective function decreases, the mutation is accepted. If the value of the objective function does not decrease, the mutation is accepted with a certain probability according to the Metroplis criterion. After repeated iterative optimization about 40,000 steps, the objective function will converge to a certain value. A new protein whose fixed motif part is similar to the target which are predicted to be stably folded is generated.

\subsection{Tool and Package Used in Protein Structure Comparison and Data Analysis}

The binding interface between SARS-CoV-2 S protein RBD and human ACE2, binding energy and residue energy decomposition are calculated using Rosetta InterfaceAnalyzer application [24]. TM score calculation is performed using tmscoring python package. Diagram and plot is generated using seaborn python package [30].

\section{ACKNOWLEDGMENTS}

This work was supported by National Natural Science Foundation of China [61431017].

\section{REFERENCES}

[1] 2021. https://covid19.who.int/

[2] "Z. Li, Y. Yi, X. Luo et al.2020. Development and clinical application of a rapid IgM-IgG combined antibody test for SARS-CoV-2 infection diagnosis. fournal of Medical Virology,92(9),1518-1524.10.1002/jmv.25727.

[3] "J. Shang, G. Ye, K. Shi et al.2020. Structural basis of receptor recognition by SARS-CoV-2. Nature,581(7807),221-+.10.1038/s41586-020-2179-y.

[4] "H. Xu, L. Zhong, J. Deng et al.2020. High expression of ACE2 receptor of 2019$\mathrm{nCoV}$ on the epithelial cells of oral mucosa. International fournal of Oral Science,12(1).10.1038/s41368-020-0074-x. 
[5] "H. Zhang, J. M. Penninger, Y. Li et al.2020. Angiotensin-converting enzyme 2 (ACE2) as a SARS-CoV-2 receptor: molecular mechanisms and potential therapeutic target. Intensive Care Medicine,46(4),586-590.10.1007/s00134-020-05985-9.

[6] "J. Zhang, H. Zeng, J. Gu et al.2020. Progress and Prospects on Vaccine Development against SARS-CoV-2. Vaccines,8(2).10.3390/vaccines8020153.

[7] "G. Zhang, S. Pomplun, A. R. Loftis et al.2020. The first-in-class peptide binder to the SARS-CoV-2 spike protein.

[8] "T. Sitthiyotha, and S. Chunsrivirot.2020. Computational Design of 25-mer Peptide Binders of SARS-CoV-2. Fournal of Physical Chemistry B,124(48),1093010942.10.1021/acs.jpcb.0c07890.

[9] "R. C. Larue, E. Xing, A. D. Kenney et al.2021. Rationally Designed ACE2-Derived Peptides Inhibit SARS-CoV-2. Bioconjugate chemistry,32(1),215223.10.1021/acs.bioconjchem.0c00664.

[10] "J. Yang, I. Anishchenko, H. Park et al.2020. Improved protein structure prediction using predicted interresidue orientations. Proceedings of the National Academy of Sciences of the United States of America,117(3),1496-1503.10.1073/pnas.1914677117.

[11] "L. Cao, I. Goreshnik, B. Coventry et al.2020. De novo design of picomolar SARSCoV-2 miniprotein inhibitors. Science,370(6515).10.1126/science.abd9909.

[12] "X. Tian, C. Li, A. Huang et al.2020. Potent binding of 2019 novel coronavirus spike protein by a SARS coronavirus-specific human monoclonal antibody. Emerging Microbes \& Infections,9(1),382-385.10.1080/22221751.2020.1729069.

[13] "M. AlQuraishi.2019. AlphaFold at CASP13. Bioinformatics,35(22),48624865.10.1093/bioinformatics/btz422.

[14] "G.-W. Wei.2019. Protein structure prediction beyond AlphaFold. Nature Machine Intelligence,1(8),336-337.10.1038/s42256-019-0086-4.

[15] "A. Madani, B. McCann, N. Naik et al.2020. ProGen: Language Modeling for Protein Generation.

[16] "M. Torrisi, G. Pollastri, and Q. Le.2020. Deep learning methods in protein structure prediction. Computational and Structural Biotechnology fournal,18(13011310.10.1016/j.csbj.2019.12.011

[17] "R. R. Eguchi, N. Anand, C. A. Choe et al.2020. IG-VAE: Generative Modeling of Immunoglobulin Proteins by Direct 3D Coordinate Generation
[18] "I. Anishchenko, T. M. Chidyausiku, S. Ovchinnikov et al.2020. De novo protein design by deep network hallucination.

[19] "C. Norn, B. I. M. Wicky, D. Juergens et al.2020. Protein sequence design by explicit energy landscape optimization.

[20] "B. V. J. A. Hoorick.2019. Image Outpainting and Harmonization using Generative Adversarial Networks.abs/1912.10960(

[21] K. Kim, Y. Yun, K.-W. Kang et al., "Painting Outside as Inside: Edge Guided Image Outpainting via Bidirectional Rearrangement with Progressive Step Learning."

[22] "Q. Xiao, G. Li, and Q. J. I. A. Chen.2020. Image Outpainting: Hallucinating Beyond the Image.8(173576-173583.

[23] "K. He, X. Zhang, S. Ren et al.2016. Deep Residual Learning for Image Recognition. 770-778.

[24] 2016. https://www.rosettacommons.org/docs/latest/application_documentation/ analysis/interface-analyzer.

[25] "B. J. Bender, A. Cisneros, A. M. Duran et al.2016. Protocols for Molecular Modeling with Rosetta3 and RosettaScripts. Biochemistry,55(34),47484763.10.1021/acs.biochem.6b00444

[26] "R. B. Cooley, J. L. Feldman, C. M. Driggers et al.2014. Structural basis of improved second-generation 3-nitro-tyrosine tRNA synthetases. Biochemistry,53(12),19161924.10.1021/bi5001239.

[27] "A. Senior, R. Evans, J. Jumper et al.2019. Protein structure prediction using multiple deep neural networks in the 13th Critical Assessment of Protein Structure Prediction (CASP13).87(1141 - 1148.

[28] "E. Wang, H. Sun, J. Wang et al.2019. End-Point Binding Free Energy Calculation with MM/PBSA and MM/GBSA: Strategies and Applications in Drug Design.

[29] "R. B. Cooley, P. A. Karplus, and R. A. Mehl.2014. Gleaning unexpected fruits from hard-won synthetases: probing principles of permissivity in non-canonical amino acid-tRNA synthetases. Chembiochem,15(12),1810-1819.10.1002/cbic.201402180.

[30] P. Lemenkova, "Python Libraries Matplotlib, Seaborn and Pandas for Visualization Geo-spatial Datasets Generated by QGIS." 